Jörg Roth-Kim

\section{Einleitung}

Die vorliegende Untersuchung will in erster Linie anhand eines Beispiels zeigen, wie Zusammenhänge, die offenbar zwischen der Stärke politischer Parteien und andern geographischen Phänomena zu bestehen scheinen, durch mathematische Formeln approximiert werden können. Meines Erachtens sind politische Parteien wie jeder andere Landschaftsinhalt geographische Phänomene.

Aus dem Ausland, namentlich aus dem anglo-amerikanischen Bereich, liegen zahlreiche Untersuchungen vor, in denen versucht wird, die Zugkraft politischer Parteien mit Gegebenheiten der Kulturlandschaft, z. B. mit Merkmalen von Bevölkerung, Siedlung oder Wirtschaft, zu korrelieren. Die Ergebnisse solcher Studien, von denen einige auch in geographischen Zeitschriften veröffentlicht worden sind, können als Grundlagen für Wahlprognosen verwendet werden.

In unserm Lande haben sich Untersuchungen dieser Art bisher hauptsächlich auf die Analyse von Wahlresultaten in Presse und amtlichen Publikationen beschränkt, in denen das Mitspielen von Faktoren außerhalb der parteipolitischen Sphäre bestenfalls am Rande und nur qualitativ vermerkt wird ${ }^{1}$. Vor kurzem konnte der Verfasser am Beispiel der Gemeinden des Bezirks Brugg (Kanton Aargau) dartun, daß auch in unsern Verhältnissen die Wahlstärke von Parteien mit andern Größen - in diesem Falle mit der Erwerbsstruktur - in Verbindung gebracht werden kann².

\section{Die politischen Parteien}

Politische Parteien stellen organisierte Gruppen der Bevölkerung dar, die sich im Aargau und in den Teilen unseres Landes, in denen das Stimm- und Wahlrecht der Frauen noch nicht Eingang gefunden hat, zusammensetzen aus männlichen Einwohnern schweizerischer Nationalität im stimm- und wahlfähigen Alter.

Im Landschaftsbild, d. h. in der Physiognomie der Landschaft, werden wir in der Schweiz kaum auf irgendwelche auffällige Elemente stoßen, welche die Existenz von Parteien verraten. Es ist aber nicht von der Hand zu weisen, daß die Parteien in der Dynamik der Landschaft eine nicht unwesentliche
Funktion innehaben. Sie vor allem nehmen sich der Meinungsbildung der Bevölkerung in öffentlichen Angelegenheiten an und suchen dem Gemeinwohl oder den Interessen bestimmter Gruppen dienende Begehren zu lancieren und durchzusetzen. Vor allem aber sind sie an der Regierung der politischen Einheiten unseres Landes maßgeblich beteiligt, sind doch die Exekutiven der Gemeinden und die Exekutiven und Legislativen unserer Kantone und der Eidgenossenschaft in ihrer großen Mehrheit durch Parteileute besetzt ${ }^{3}$, welche in ihren Aktionen oft durch die von ihren Parteien vertretenen Interessen, Programme, Denkweisen und Weltanschauungen beeinflußt sind.

Darüber hinaus bilden die Parteien "Keime», an denen sich etwa parteipolitisch orientierte Jugendund Frauengruppen, Kultur- und Sportvereine und Presseorgane «kristallisieren».

$\mathrm{Da}$ die meisten Parteien mehr oder weniger profiliert die Interessen gewisser sozialer, wirtschaftlicher oder konfessioneller Gruppen wahrnehmen, sollten sich Zusammenhänge zwischen der parteipolitischen Struktur und der sozialen, wirtschaftlichen und konfessionellen Gliederung der Bevölkerung eines Gebietes zeigen. Solche Relationen konnten für die Gemeinden des Bezirks Brugg nachgewiesen werden².

\section{Problemstellung}

Lassen sich nun aber auch in den übrigen Teilen des Kantons Aargau Beziehungen zwischen der Stärke einer Partei und Werten irgendwelcher anderer Größen feststellen? Zur Beantwortung dieser Frage wird versucht, die Resultate der Nationalratswahlen von 1963 einigen Daten aus der eidgenössischen Volkszählung von 1960 gegenüberzustellen 4 .

\section{Stärke der Parteien}

In unserem Lande wird der Bestand an eingeschriebenen Mitgliedern der einzelnen Parteien kaum bekanntgemacht. Deren zahlenmäßige Bedeutung läßt sich deshalb nur durch Auswertung von Wahlresultaten ermitteln. Hiefür kann man entweder die Anzahl der in die Urne gelegten Parteiwahlzettel 
(Kandidatenlisten, welche die Bezeichnung einer Partei tragen) oder aber die Parteistimmenzahlen (jeder auf einem Parteiwahlzettel aufgeführte Name eines Kandidaten zählt eine Parteistimme) verwenden. Für unsere Studie kommt nur die zweite Methode in Betracht, da die Anzahl der Parteiwahlzettel für Bezirke nicht ausgewiesen wird5.

Figur 1 vermittelt ein grobes Bild über die Gliederung der Parteistimmen im Aargau anläßlich der Nationalratswahlen 1963. Auf Bezirksebene präsentieren sich als stärkste Parteien mit relativem Mehr der Parteistimmen einerseits die Sozialdemokratische Partei, die in den stark industrialisierten und überwiegend protestantischen Bezirken Aarau, Brugg, Kulm, Lenzburg und Zofingen dominiert, und die Konservativ-christlichsoziale Volkspartei, die in den übrigen, mehrheitlich katholischen Bezirken (Baden, Bremgarten, Laufenburg, Muri, Rheinfelden, Zurzach) die Spitze hält.

\section{Korrelation der Parteistimmen mit andern Größen}

Die Betrachtung der Figur 1 läßt gewisse Zusammenhänge zwischen der Parteistimmengliederung und der Erwerbs- oder der konfessionellen Struktur der Bevölkerung vermuten.

Wir gliedern deshalb vorerst die Wahlberechtigten nach dem Merkmal «Erwerb» in die Klassen

$\mathrm{x}=$ hauptberuflich Berufstätige im primären Wirtschaftssektor (Land- und Forstwirtschaft);

$\mathrm{y}=$ hauptberuflich Berufstätige im sekundären Wirtschaftssektor (Industrie, Handwerk, Baugewerbe);

$\mathrm{z}=$ hauptberuflich Berufstätige im tertiären Wirtschaftssektor (übrige Erwerbszweige);

$\mathrm{n}=$ nichtberufstätige Wahlberechtigte;

und innerhalb jeder dieser Klassen nach dem Merkmal «Konfession» in

$\mathrm{x}_{\mathrm{v}}, \mathrm{y}_{\mathrm{v}}=$ römisch-katholische Wahlberechtigte der betreffenden Klasse;

$\mathrm{x}_{\mathrm{w}}, \mathrm{y}_{\mathrm{w}}$ usw. = Wahlberechtigte anderer oder keiner Konfession der betreffenden Klasse. (Diese mit ${ }_{w}$ indizierten Unterklassen bestehen überwiegend aus Protestanten.)

Um die Bezirke miteinander vergleichen zu können; legen wir fest, daß x, y usw. in Prozenten des jeweiligen Bezirkstotals an Wahlberechtigten $(=t)$ ausgedrückt werden sollen. Nun möchten wir Gruppen von Wählern mit bestimmten Merkmalen oder Merkmalskombinationen korrellieren mit den Parteistimmenanteilen einzelner Parteien, müssen aber

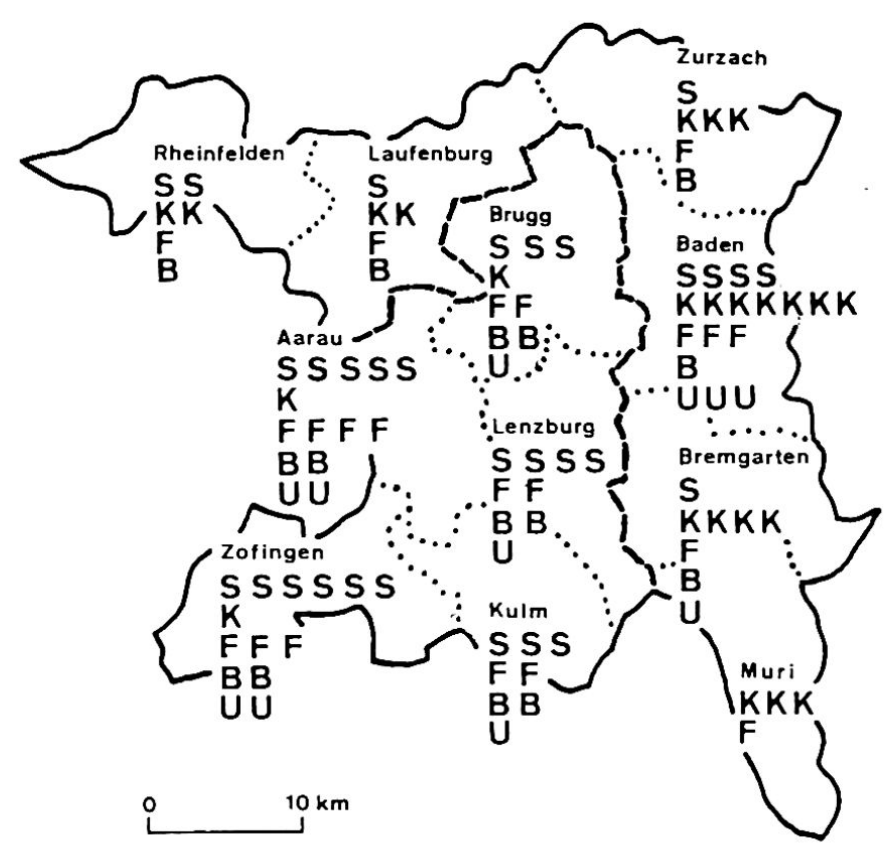

Figur 1. Nationalratswahlen 1963: Gliederung der im Kanton Aargau abgegebenen Parteistimmen nach Bezirken und Parteien.

1 Signatur (Buchstabe) $=10000$ Parteistimmen $=$ rund $750-800$ Wähler a)

$\mathrm{S}=$ Sozialdemokratische Partei, $\mathrm{K}=$ Konservativ-christlichsoziale Volkspartei, $\mathrm{F}=$ Freisinnige Partei, B = Bauern-, Gewerbe- und Bürgerpartei, $\mathrm{U}=$ Ubrige Parteien und Listen.

Ausgezogene Linie $=$ Grenze des Kantons Aargau Gestrichelte Linie = Bezirksgrenzen, welche zugleich die Konfessionsgrenze bilden $b$ )

Punktierte Linien = Übrige Bezirksgrenzen

Quellen: a) Nationalratswahlen 1963, p. 123;

b) Eidg. Volkszählung 1960, Band 2, p. 52-59.

feststellen, daß die Volkszählungspublikationen keine Angaben über die Gliederung der Wahlberechtigten nach Erwerb und Konfession enthalten. Es bleibt uns deshalb nichts anderes übrig, als die Variablen $\mathrm{x}$, y usw. durch entsprechende Hilfsvariablen X, Y usw. zu ersetzen, die in den Volkszählungsbänden figurieren und von denen wir annehmen, daß ihre Werte gute Annäherungen an die unbekannten Werte von x, y usw. darstellen. Die im folgenden berücksichtigten Größen X, Y usw. und deren Abkürzungen sind in der Legende zu Figur 2 aufgeführt 6 .

Um die vorliegende Arbeit nicht zu umfangreich werden zu lassen, wird die Untersuchung nur auf Bezirksebene durchgeführt und auf die vier größten Parteien des Kantons beschränkt ${ }^{7}$ : die Sozialdemokratische Partei, die Konservativ-christlichsoziale Volkspartei (bis ca. 1955: Katholisch-konservative Partei), die Freisinnige Partei und die Bauern-, Gewerbe- und Bürgerpartei. Hinter diesen Parteien scharten sich bei den Nationalratswahlen des Jahres 1963 in den elf aargauischen Bezirken zwischen 83 und $97 \%$ aller Wähler. 

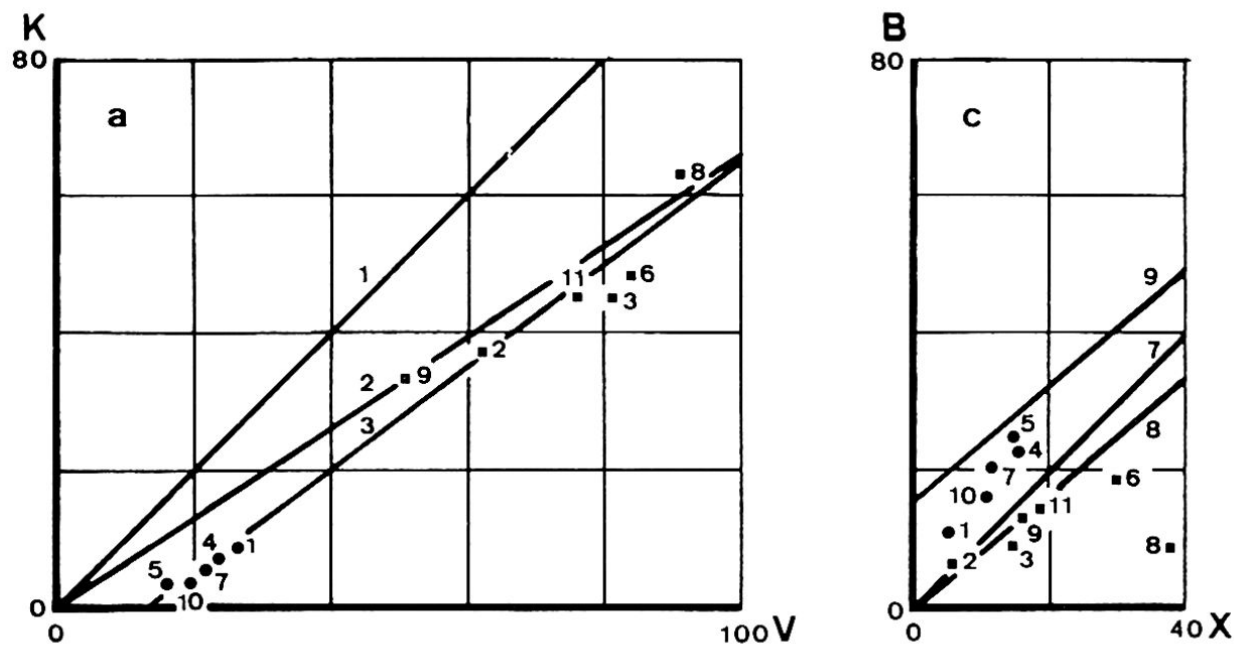

Figur $2 \mathrm{a}-\mathrm{d}$.

Beziehungen zwischen

Konfession, Erwerb und

Parteistimmen in den elf

Bezirken des Kantons

Aargau 1960/1963
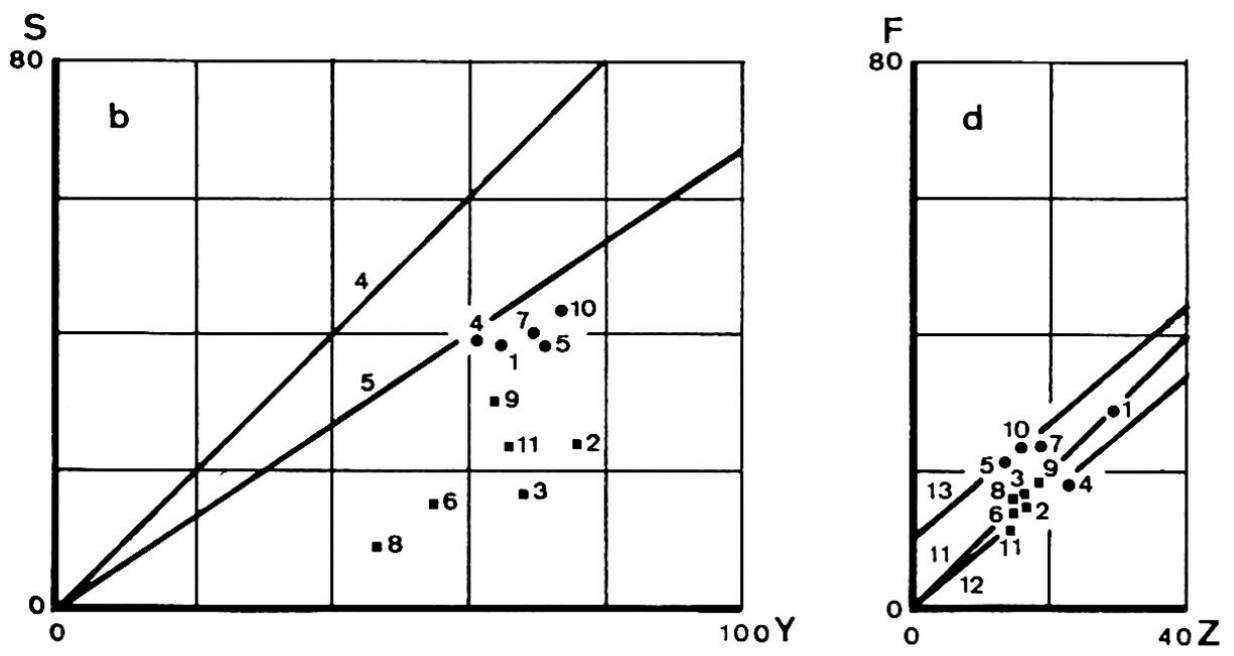

$\mathrm{B}=$ Anteil der Bauern-, Gewerbe- und Bürgerpartei (BGB)

$\mathrm{F}=$ Anteil der Freisinnigen Partei

$\mathrm{K}=$ Anteil der Konservativ-christlichsozialen Volkspartei

$\mathrm{S}=$ Anteil der Sozialdemokratischen Partei

$\mathrm{V}=$ Anteil der Römisch-Katholiken

$\mathrm{W}=$ Anteil der Einwohner anderer oder keiner Konfession

$\mathrm{X}=$ Anteil der im primären Wirtschaftssektor (Land-, Forstwirtschaft)

$\mathrm{Y}=$ Anteil der im sekundären Wirtschaftssektor (Industrie, Handwerk, Baugewerbe)

$\mathrm{Z}=$ Anteil der im tertiären Wirtschaftssektor (übrige Erwerbszweige)

am Total der in einem Bezirk bei den Nationalratswahlen 1963

abgegebenen Parteistimmen, in Prozent a)

an der Wohnbevölkerung eines Bezirks im Jahre 1960, in Prozent b)

beschäftigten Berufstätigen am Total der in einem Bezirk im Jahre 1960 wohnhaften männlichen Berufstätigen, in Prozent b)

Aus den Definitionen ergibt sich: $\mathrm{V}+\mathrm{W}=100, \mathrm{X}+\mathrm{Y}+\mathrm{Z}=100$

Kreisscheibensignaturen $=$ Bezirke mit überwiegend nichtkatholischer $(\approx$ protestantischer $)$ Wohnbevölkerung im Jahre 1960 b)

Quadratsignaturen = Bezirke mit überwiegend römisch-katholischer Wohnbevölkerung im Jahre 1960 b)

Zur Identifikation sind die Signaturen der Bezirke mit Nummern versehen: 1 Aarau, 2 Baden, 3 Bremgarten, 4 Brugg, 5 Kulm, 6 Laufenburg, 7 Lenzburg, 8 Muri, 9 Rheinfelden, 10 Zofingen, 11 Zurzach.

Jede eingezeichnete Gerade trägt die Nummer der ihr entsprechenden im Text aufgeführten Gleichung.

Quellen: a) Nationalratswahlen 1963, p. 123; b) Eidg.Volkszählung 1960, Band 2, p. 52-59. Siehe ferner Anmerkung 6 am Schluß des Textteils.

Die Aufgabe soll zudem vereinfacht werden, indem wir

1. annehmen, daß die persönlichen und fachlichen Qualitäten der Kandidaten aller Parteien gleich waren;
2. weiter annehmen, daß alle Stimmberechtigten zur Urne gingen 8 ;

3. verzichten auf verfeinerte, jedoch aufwendige statistische Untersuchungsverfahren (wie z. B. Mehrfachkorrelation oder Faktorenanalyse ${ }^{9}$ ) und 
dafür versuchen wollen, mittels einfacher Approximationsmethoden $\mathrm{zu}$ brauchbaren mathematischen Modellen zu gelangen.

Konservativ-christlichsoziale Volkspartei. Es liegt nahe, den Anteil an Stimmen, den diese Partei in den einzelnen Bezirken buchen konnte, mit dem Prozentsatz der Römisch-Katholiken unter der Wählerschaft zu vergleichen (Fig. 2a). Offensichtlich variieren die Werte der beiden Größen nicht Zufällig miteinander, sondern stehen in einem $\mathrm{Zu}$ sammenhang. Um ein mathematisches Modell für diese Beziehung zu erhalten, suchen wir eine Funktion, die dem beobachteten Punkteschwarm möglichst gut angepaßt ist ${ }^{10}$.

In einem ersten Approximationsschritt nehmen wir an, da $\beta$ jeder katholische Wähler der Konservativchristlichsozialen Partei stimmte, daß also

$$
\mathrm{K}=\mathrm{V}
$$

sei. Diese Funktion ergibt jedoch viel zu hohe KWerte. Offenbar stimmte nur ein Teil der Katholiken den Konservativen ${ }^{11}$. Tatsächlich erreichten diese selbst in einem Bezirk wie Muri mit rund 90\% katholischen Wählern nur etwa $60 \%$ der Parteistimmen. Als zweite Annäherung setzen wir deshalb

$$
\mathrm{K}=\frac{2}{3} \mathrm{~V} \text {. }
$$

Ein Vergleich dieser Geraden mit der effektiven Punkteverteilung ergibt eine auffällige Divergenz aller überwiegend protestantischen Bezirke. Vermutlich ist hier die konservative Parteiorganisation eher schwach, so daß ein größerer Prozentsatz der Katholiken (z. B. Fabrikarbeiter) andere Parteien begünstigt. Wir wollen den «erodierenden» Einfluß der industrialisierten protestantischen Umgebung auf die katholischen Wähler berücksichtigen in Form eines Korrekturgliedes in unserer Funktion, dessen ungefähre mittlere Größe sich durch einige Versuche mit dem Zahlenmaterial mit - 0,10 W bestimmen läßt. Dies führt im dritten Approximationsschritt zu

$$
\mathrm{K}=\frac{2}{3} \mathrm{~V}-0,10 \mathrm{~W} .
$$

Nun ist aber $\mathrm{V}+\mathrm{W}=100$. Daraus folgt

$\mathrm{K}=\frac{2}{3} \mathrm{~V}-0,10(100-\mathrm{V})=\frac{23}{30} \mathrm{~V}-10$.

Da $\frac{23}{30} \approx \frac{3}{4}$ schreiben wir

$$
\mathrm{K}=\frac{3}{4} \mathrm{~V}-10 .
$$

Damit erhalten wir eine gute, durch einen ChiQuadrat-Test gesicherte Annäherung an den Punk- teschwarm. Die nach dieser Funktion berechneten (theoretischen) K-Werte weichen von den effektiven (beobachteten) Werten höchstens um 5,8 Prozentpunkte (Bez. Bremgarten 50,8 statt 45,0\%).

Sozialdemokratische Partei. Es ist bekannt, daß sich die sozialdemokratischen Wähler vorwiegend aus der Industriearbeiterschaft rekrutieren. Wir setzen deshalb den Parteistimmenanteil der Sozialdemokraten in Beziehung zum Anteil, mit welchem die im sekundären Wirtschaftssektor berufstätigen Männer an der gesamten aktiven männlichen Wohnbevölkerung eines Bezirks beteiligt waren (Fig. 2b).

Eine erste Annäherung

$$
\mathrm{S}=\mathrm{Y}
$$

vermag nicht zu genügen. Wir müssen nämlich in Rechnung stellen, daß 1960 im Aargau 32\% der sekundären männlichen Berufstätigen nicht wahlberechtigt waren (Ausländer 23\%, Schweizer unter 20 Jahren etwa 9\%)12: Ferner ist zu berücksichtigen, daß gewisse Gruppen sekundärer Berufstätiger, z. B. selbständige Handwerker, Industrieunternehmer und Industriebüropersonal, in ihrer großen Mehrheit kaum die "rote» Liste einlegen. Dafür erhalten die Sozialisten gewöhnlich Schützenhilfe von Berufstätigen aus andern Wirtschaftssektoren (z. B. SBB- und PTT-Arbeitskräfte) und von nichtberufstätigen Wählern (z. B. pensionierten Arbeitern). Da aber über solche Wählerverlagerungen keine Zahlen vorliegen, nehmen wir vorderhand an, daß

$$
\mathrm{S}=\frac{2}{3} \mathrm{Y}
$$

sei. Von dieser Funktion weichen die S-Werte der protestantischen Bezirke wenig, jene der katholischen Bezirke jedoch stark ab, da offenbar hier ein ansehnlicher Teil der katholischen Arbeiterschaft nicht sozialistisch wählt. Der mittlere Einfluß des konfessionellen Faktors kann durch ein Korrekturglied von $-0,30 \mathrm{~V}$ angenähert werden.

Damit erhalten wir mit

$$
\mathrm{S}=\frac{2}{3} \mathrm{Y}-0,30 \mathrm{~V}
$$

eine durch Chi-Quadrat-Prüfung gesicherte Approximation, die einzig im Falle des Bezirks Baden eine unbefriedigend große Differenz (7,7 Prozentpunkte) zwischen beobachtetem und theoretischem Wert ergibt $(24,4$ bzw. $32,1 \%)$.

Bauern-, Gewerbe- und Bürgerpartei. Diese Partei findet im allgemeinen den Hauptharst ihrer Wähler unter den Berufstätigen des primären Sektors. Auch hier entspricht

$$
\mathrm{B}=\mathrm{X}
$$

nicht im gewünschten $\mathrm{Maß}$ der vorliegenden Punk- 
teverteilung (Fig. 2c). Da 1960 rund 15\% der primären Berufstätigen nicht wahlberechtigt waren (Ausländer $6 \%$, Schweizer unter 20 Jahren etwa $9 \%)^{12}$, setzen wir

$$
\mathrm{B}=0,85 \mathrm{X} \text {. }
$$

Nun erhält jedoch die Bauern-, Gewerbe- und Bürgerpartei aus Kreisen des Kleingewerbes (selbständige Handwerker, Detailhändler usw.) einen ansehnlichen Zuzug, der (unabhängig von X) mit 15 Prozentpunkten angenommen wird:

$$
\mathrm{B}=0,85 \mathrm{X}+15 \text {. }
$$

Anderseits muß damit gerechnet werden, daß sich ein Teil der Wähler aus dem primären Sektor nicht hinter die BGB-Liste stellt, und zwar vor allem aus konfessionellen Gründen. Der Verlust an katholischen Stimmen kann durch $-0,25 \mathrm{~V}$ ausgedrückt werden. Somit ergibt sich

$$
\mathrm{B}=0,85 \mathrm{X}+15-0,25 \mathrm{~V} \text {. }
$$

Wenn wir die nach dieser Funktion errechneten Werte mit den tatsächlichen B-Werten vergleichen, finden wir in 10 Bezirken Abweichungen von höchstens 4,0 Prozentpunkten. Einzig der Bezirk Muri erweist sich mit theoretisch $24,3 \%$ statt effektiv $9,2 \%$ als "Ausreißer». Nur wenn wir diesen Fall ausschließen, kann die Annehmbarkeit des Modells für die übrigen Bezirke als gesichert gelten.

Freisinnige Partei. Auf Grund der bisherigen Untersuchungen, die den primären Sektor mit der BGB und den sekundären mit den Sozialdemokraten in Verbindung gebracht haben, drängt es sich auf, die männlichen Berufstätigen im tertiären oder Dienstleistungssektor mit den Parteistimmen der Freisinnigen zu korrelieren (Fig. 2d). Obwohl

$$
\mathrm{F}=\mathrm{Z}
$$

auf den ersten Blick keine schlechte Annäherung an die beobachteten Werte darstellt, ist doch auch hier der Anteil der Nichtwahlberechtigten (Ausländer $6 \%$, Schweizer unter 20 Jahren etwa $9 \%$, insgesamt $15 \%)^{12}$ zu berücksichtigen, also

$$
\mathrm{F}=0,85 \mathrm{Z} \text {. }
$$

Da diese Funktion für mehr als die Hälfte der Bezirke zu tiefe Werte ergibt, müssen wir annehmen, daß der Freisinn von Wählern außerhalb des tertiären Sektors Zuzug erhält. Die Wirkung dieser Wählerverlagerung wird auf 10 Prozentpunkte geschätzt:

$$
\mathrm{F}=0,85 \mathrm{Z}+10 .
$$

Wie schon zuvor muß auch hier noch eine durch Versuche ermittelte Korrektur angefügt werden, die den Abgang katholischer Wähler zum Ausdruck bringt:

$$
\begin{aligned}
& F=0,85 Z+10-(3+0,07 V) \\
& F=0,85 Z+7-0,07 V .
\end{aligned}
$$

Auch in diesem Fall zeitigt die Formel gute Úbereinstimmung mit den tatsächlichen Werten. Wenn auch im Bezirk Brugg der Unterschied zwischen effektivem und theoretischem F-Wert $(18,5 \%$ bzw. $25,1 \%$ ) etwas groß ist, so darf doch auf Grund des Chi-Quadrat-Tests die Gültigkeit des Modells als gesichert angesprochen werden.

Interessant ist, daß in der obigen Formel der Faktor von $\mathrm{V}$ mit dem Wert 0,07 erheblich kleiner ist als in den entsprechenden Formeln für $S$ und B $(0,30$ bzw. 0,25). Dies läßt vermuten, daß die Freisinnige Partei die geringsten, die Sozialdemokraten hingegen in ihrem potentiellen "Machtbereich" - der Wählerschaft des tertiären bzw. des sekundären Sektors - die größten Einbußen zu verzeichnen hatten, welche durch Abwanderung von Katholiken zu andern Parteien (sehr wahrscheinlich zur Hauptsache zur Konservativ-christlichsozialen Volkspartei) verursacht wurden.

Gültigkeit der Modelle. Eine praktische Anwendung dürften die oben aufgestellten Formeln dann finden, wenn sie zur Aufstellung von Wahlprognosen herbeigezogen werden könnten. Dies würde allerdings bedingen, daß die anhand der Stichprobe der elf aargauischen Bezirke und für das Stichjahr 1963 festgestellten Beziehungen über längere Zeit und womöglich über ein größeres Gebiet ihre Gültigkeit behalten müßten. Die Anwendung der Formeln auf die Nachbarkantone (Zahlen 1960/ 1963) ergab mehrheitlich akzeptable Ergebnisse. Auf eine mehr als nur momentane Validität wird man jedoch erst schließen dürfen, wenn die Resultate der Nationalratswahlen von 1971 mit Werten verglichen werden können, die nach unsern Formeln auf Grund von Daten aus der eidgenössischen Volkszählung 1970 berechnet wurden.

\section{Zusammenfassung}

Die vorliegende Untersuchung zeigt am Beispiel der Resultate der Nationalratswahlen von 1963 im Kanton Aargau, daß die Stärke der vier größten aargauischen Parteien in den einzelnen Bezirken mathematisch mit der Erwerbsstruktur und den konfessionellen Verhältnissen in Beziehung gebracht werden kann. Es zeigt sich auch, daß der Geograph ohne komplizierte mathematisch-statistische Techniken und ohne Computer bereits durch einfache Approximationsmethoden, die als Hilfsmittel lediglich eine Rechenmaschine oder notfalls einen $\mathrm{Re}$ chenschieber erfordern, zu zwar rohen, aber durchaus brauchbaren gesicherten mathematischen Mo- 
dellen gelangen kann, die teilweise auch in Nachbarkantonen Gültigkeit haben und unter Umständen in der Erstellung von Wahlprognosen praktische Anwendung finden dürften.

\section{Anmerkungen und Quellen}

1 Zum Beispiel: Nationalratswahlen 1963. Eidgenössisches Statistisches Amt. Bern, 1965. Beiträge zur schweizerischen Statistik, Heft 34, p. 27-30, $49,53$.

2 Roth, Jörg: Zur Kulturgeographie des Bezirks Brugg (Kanton Aargau). Untersiggenthal: HarderDruck, 1968. Diss. ETH, Zürich, Nr. 4187, p. 232 -235 .

3 Vergleiche Nationalratswahlen 1963, p. 198.

4 Die Ergebnisse der jüngsten Nationalratswahlen (von 1967) eignen sich wegen ihres großen zeitlichen Abstandes zur letzten Volkszählung (1960) schlecht für unsere Untersuchung. Die Nationalratswahlen von 1959 hätten zwar der genannten Volkszählung noch näher gelegen, scheinen aber dem Verfasser zu weit zurückzuliegen, um noch aktuell zu sein.

5 Wenn nun hier die Stärke einer Partei an der Anzahl der erhaltenen Kandidatenstimmen gemessen wird, so ist dabei zu beachten, daß diese Stimmen in starker Mehrheit von Sympathisanten außerhalb der Parteiorganisation und nur zu einem kleinen Teil von eigentlichen Parteimitgliedern stammen müssen, da der Anteil der letzteren an der Wählerschaft im Durchschnitt aller Parteien nach einer Schätzung des Eidgenössischen Statistischen Amtes nur etwa 15 bis $20 \%$ beträgt (Nationalratswahlen 1963, p. 23).

\begin{tabular}{lrr} 
Primärer Sektor & $\mathrm{X}=13,2 \%$ & $15,1 \%$ \\
Sekundärer Sektor & $\mathrm{Y}=68,1 \%$ & $63,5 \%$ \\
Tertiärer Sektor & $\mathrm{Z}=18,7 \%$ & $21,4 \%$ \\
Nichtberufstätige & - & - \\
\cline { 2 - 2 } & $100,0 \%$ & $100,0 \%$
\end{tabular}

Die größte Abweichung zwischen Hilfsvariablenund Variablenwert weist der sekundäre Sektor auf, wo infolge des großen Ausländeranteils einerseits und der notwendigen Mitberücksichtigung der nichtberufstätigen Wahlberechtigten anderseits der Fehler rund 11 Prozentpunkte beträgt. Weil für Bezirke nur die Erwerbsstruktur des Totals der männlichen Berufstätigen aus den Volkszählungsbänden ermittelt werden kann, müssen wir versuchen, diese Fehler durch entsprechende Korrekturfaktoren und -glieder in unsern Formeln auszugleichen.
6 Grundlagen (außer dem bereits zitierten Band Nationalratswahlen 1963): Eidgenössische Volkszählung 1960. Eidg. Statistisches Amt. Band 2: Kanton Aargau. Bern 1964. Stat. Quellenwerke der Schweiz, Heft 367. Band 27: Schweiz I/Geschlecht, Heimat ... Bern 1964. Stat. Quellenwerke der Schweiz, Heft 366. Band 28: Schweiz II/Erwerb und Beruf. Bern 1965. Stat. Quellenwerke der Schweiz, Heft 385.

An dieser Stelle scheint es angezeigt, einige Überlegungen über die Größenordnung der Fehler anzustellen, die wir akzeptieren müssen, wenn wir statt der unbekannten Werte der Größen x, y... die Werte der Hilfsvariablen X, Y ... verwenden.

Bei der Substitution von $\mathrm{X}, \mathrm{y}$ und $\mathrm{z}$ durch $\mathrm{X}, \mathrm{Y}$ und $\mathrm{Z}$ ist zu beachten, daß die Elemente der Grundgesamtheit "Wahlberechtigte» nur teilweise identisch sind mit den Elementen der Grundgesamtheit «Männliche Berufstätige». Im Kanton Aargau standen im Jahre 1960 die beiden Grundgesamtheiten wie folgt zueinander:

Männliche Berufstätige total 120519

Berufstätige Ausländer 99306

Berufstätige Schweizer unter 20 Jahren -10704

Berufstätige Wahlberechtigte $\quad 88602$

Nichtberufstätige Wahlberechtigte $\quad+\quad 9087$

Wahlberechtigte total $=$ Schweizer im

Alter von 20 Jahren und mehr

97689

(Zahlen nach Eidg. Volkszählung, Band 2, p. 49, 104, 146.)

Die Berechnung (nach op. cit., p. 49, 104, 108, 146) bzw. Schätzung der Erwerbsstruktur der einzelnen Grundgesamtheiten ergibt folgendes Bild:

\begin{tabular}{|c|c|}
\hline $\begin{array}{l}\text { Berufstätige } \\
\text { Wahlberechtigte }\end{array}$ & $\begin{array}{l}\text { Wahlberechtigte } \\
\text { total }\end{array}$ \\
\hline rund $16 \%$ & $\mathrm{x}=$ rund $15 \%$ \\
\hline rund $63 \%$ & $\mathrm{y}=\operatorname{rund} 57 \%$ \\
\hline rund $21 \%$ & $\mathrm{z}=$ rund $19 \%$ \\
\hline - & $\mathrm{n}=\quad 9 \%$ \\
\hline $100 \%$ & $100 \%$ \\
\hline
\end{tabular}

Da die konfessionelle Gliederung der Wahlberechtigten wie der Berufstätigen in den amtlichen Publikationen nicht figuriert, machen wir die Annahme, daß die Katholiken (und entsprechend auch die Nichtkatholiken) in der gesamten Wohnbevölkerung, im Total der Wahlberechtigten und innerhalb der einzelnen Erwerbsklassen der Wahlberechtigten $(\mathrm{x}, \mathrm{y}, \mathrm{z}, \mathrm{n})$ mit annähernd gleichem Prozentsatz vertreten seien, daß also

$$
\frac{\mathrm{v}}{\mathrm{t}}=\frac{\mathrm{x}_{\mathrm{v}}}{\mathrm{x}}=\frac{\mathrm{y}_{\mathrm{r}}}{\mathrm{y}}=\frac{\mathrm{z}_{\mathrm{v}}}{\mathrm{z}}=\frac{\mathrm{n}_{\mathrm{v}}}{\mathrm{n}}=\mathrm{V} .
$$


Nun betrug aber 1960 im Kanton Aargau der Anteil der Römisch-Katholischen an der

- Gesamtbevölkerung

- Bevölkerung schweizerischer Nationalität (und beiderlei Geschlechts)

- männlichen Bevölkerung schweizerischer Nationalität

- Gesamtheit der Wahlberechtigten

$$
40,1 \%
$$$$
\text { ca. } 40 \%
$$

Die Abweichung in der konfessionellen Struktur der Schweizer von der Gesamtbevölkerung rührt davón her, daß rund $20 \%$ der Katholiken beiderlei Geschlechts (aber nur 3\% der Nichtkatholiken) Ausländer waren. Wir müssen also in Kauf nehmen, $\mathrm{da} ß$ unsere $\mathrm{V}$-Werte gegenüber den wirklichen Verhältnissen sehr wahrscheinlich im Mittel um rund 5-6 Prozentpunkte zu hoch sind, wobei dieser Fehler um so größer ist, je höher der Ausländeranteil an der Wohnbevölkerung einer Region steht. Der Verfasser verzichtete jedoch bewußt darauf, den Anteil der Ausländer als weitere Variable in die Untersuchung einzuführen. (Zitierte Prozentwerte berechnet nach Eidg. Volkszählung, Band 2, p. 31, 49.)
7 Aufzählung nach abnehmender Anzahl der 1963 erhaltenen Parteistimmen (siehe Nationalratswahlen 1963, p. 123).

8 Im Aargau, einem Kanton mit Stimmzwang, betrug 1963 die Wahlbeteiligung 83\% (Ibid., p. 14).

9 Vergleiche z. B. Steiner, Dieter: Die Faktorenanalyse ... In: Geographica Helvetica, Vol. 20, 1965 , p. $20-34$.

10 Eine solche Funktion würde im vorliegenden Fall der Punkteverteilung (Fig. 2a) normalerweise durch Berechnung der Gleichung für die Regressionsgerade bestimmt, was $\mathrm{K}=0,73 \mathrm{~V}-9,3$ ergäbe (Einheit: Prozent der Wählerschaft). Der Verfasser möchte hier jedoch ein zwar gröberes, aber einfacheres Approximationsverfahren demonstrieren, das auch in den Fällen der andern Parteien, wo zwei unabhängige Variable berücksichtigt werden, anwendbar ist.

11 Vergleiche hiezu Nationalratswahlen 1963, p. 49, 53.

12 Berechnung bzw. Schätzung der Nichtwahlberechtigten in den drei Wirtschaftssektoren auf Grund der Daten in Eidg. Volkszählung 1960, Band 2, p. 104, 108, 146.

\title{
Fremdenverkehr und Uferschutz an den großen Voralpenseen
}

\author{
Erich Schwabe
}

Die nachstehende kleine Studie setzt sich zum Ziel, überblicksweise nachzuprüfen, in welchem Umfang und in welcher Art der Fremdenverkehr das Landschaftsbild im unmittelbaren Uferbereich einiger unserer großen Voralpen- und Alpenrandseen bestimmt und wie weit einzelne landschaftsschützerische Maßnahmen eine unschöne bauliche Weiterentwicklung allenfalls $\mathrm{zu}$ verhindern imstande wären. Unser verehrter Jubilar hat, wie aus andern Beiträgen dieses Heftes hervorgeht, neben der Erforschung und Planung der Landschaft auch die Fragen des Landschaftsschutzes nie vernachlässigt; als kompetenter Vertreter der landesplanerischen Kreise gehörte er übrigens während einer Reihe von Jahren dem Zentralvorstand des Schweizer Heimatschutzes an.
Unsere Darlegungen widmen sich der Umgebung sechs großer, tief in den Alpenkörper eingesenkter, zum Teil aus ihm heraus ins Alpenvorland tretender Seebecken. Schroffe, von den eiszeitlichen Gletschern zuweilen zu blanken Felswänden geschliffene oder von dichtem Wald bestandene Ufer prägen in weiten Partien ihren Charakter und schaffen, zusammen mit den sie überragenden Gebirgsstöcken, eine Naturszenerie, deren Schönheit, etwa auch Wildheit, stets von neuem bewundert wird und nicht von ungefähr Bestrebungen zu dauerndem Schutze ausgelöst hat. Den Steilhängen, die in gemilderter Form, waldüberwachsen und in viel geringere Höhen wuchtend, auch im Alpenvorland erscheinen - dort etwa von Molassefelsen gebildet -, treten flachere Uferstreifen zur Seite; lieblichere 